EPJ manuscript No.

(will be inserted by the editor)

\title{
Vacuum polarization in muonic and antiprotonic atoms: the fine structure at medium $Z$
}

\author{
E. Yu. Korzinin ${ }^{1}$, V. G. Ivanov ${ }^{1,2}$, and S. G. Karshenboim ${ }^{1,3 a}$ \\ 1 D. I. Mendeleev Institute for Metrology (VNIIM), 190005, St. Petersburg, Russia \\ ' 2 Pulkovo Observatory, 196140, St. Petersburg, Russia \\ 3 Max-Planck-Institut für Quantenoptik, 85748, Garching, Germany
}

Received: date / Revised version: date

\begin{abstract}
Effects of vacuum polarization modify the energy levels in atoms with an orbiting particle heavier than an electron. The dominant effect is due to the Uehling potential. In this paper we consider the relativistic corrections to the energy levels caused by the Uehling potential and in particular the fine structure in muonic and antiprotonic atoms. We derive general expressions and consider in detail specific regions of parameters which allow simple asymptotic expansion. We take into account the recoil effects and anomalous magnetic moment in the case of an antiproton as the orbiting particle.
\end{abstract}

PACS. 36.10.Gv Mesonic atoms and molecules, hyperonic atoms and molecules - 31.30.Jv Relativistic and quantum electrodynamic effects in atoms and molecules - 32.10.Fn Fine and hyperfine structure

\section{Introduction}

Quantum electrodynamics (QED) corrections to the energy levels of bound states have very different structure from one atom to another. The dominant QED effect for exotic atoms with an orbiting particle heavier than an electron is due to the so-called Uehling potential, which is caused by a free electron vacuum polarization loop. In a previous paper [1] we studied the nonrelativistic effects which are in particular responsible for the Lamb shift.

In principle, the results were known for a few low-lying levels for a while [2] however, they are rather complicated and the aim of [1] was to derive simple expressions for arbitrary states, which may be realized as certain asymptotic expansions. For instance, the expansions are possible over a small parameter $Z \alpha$ (where $Z$ is the nuclear charge) and a large parameter $Z \alpha m / n^{2} m_{e}$ (where $m$ is the mass of an orbiting particle and $n$ is the principal quantum number). Some other expansions are also possible.

Analysis in 1] was performed in the leading non-relativistic approximation. Here we consider the first relativistic correction. This correction is the dominant QED contribution to the fine structure and we pay special attention to the fine structure effects in muonic and antiprotonic atoms. Certain nonrelativistic asymptotics were previously studied in 4,5], while in [1] we considered a specific case of high $n$ which may be of particular interest due to antiprotonic atoms.

Here we derive a general expression for the dominant correction to the Lamb shift at a medium value of the

\footnotetext{
a E-mail: sek@mpq.mpg.de
}

nuclear charge $Z$. This correction is $j$-independent and we also found a relativistic correction which depends on the angular momentum $j$. Finally, the vacuum polarization corrections are presented in the form

$$
\begin{gathered}
\Delta E\left(n l_{j}\right)= \\
\frac{\alpha}{\pi}(Z \alpha)^{2} \frac{m c^{2}}{n^{2}}\left[F_{n l}\left(\kappa_{n}\right)+(Z \alpha)^{2} H_{n l j}\left(\kappa_{n}\right)+\ldots\right],
\end{gathered}
$$

where

$$
\kappa_{n}=\frac{\kappa}{n}=\frac{1}{n} \frac{Z \alpha m}{m_{e}}
$$

The first term (the $F$-term) is responsible for most of the Lamb shift and was already studied in detail in 11. The relativistic correction (the $H$-term) is a small correction to the Lamb shift, but it is responsible for the dominant radiative contribution to the fine structure, which we define here as splitting for the case of $j \neq j^{\prime}$ while $n=n^{\prime}$ and $l=l^{\prime}$. Due to that we consider in detail the fine-structure difference

$$
\Delta_{n l}^{\mathrm{FS}}\left(\kappa_{n}\right)=H_{n, l, l+1 / 2}\left(\kappa_{n}\right)-H_{n, l, l-1 / 2}\left(\kappa_{n}\right) .
$$

In the atoms of interest the mass $m$ and parameter $\kappa_{n}$ can be $m \simeq 207 m_{e}$ and $\kappa_{n} \simeq 1.5 Z / n$ (in a muonic atom) and $m \simeq 1836 m_{e}$ and $\kappa_{n} \simeq 13.4 Z / n$ (in an antiprotonic atom). 
E. Yu. Korzinin, V. G. Ivanov, and S. G. Karshenboim: Vacuum polarization...

\section{The relativistic expression for the Uehling term and the fine structure}

An exact relativistic expression for the Uehling correction to the energy is of the form

$$
\Delta E\left(n l_{j}\right)=\int d r r^{2}\left(\left|f_{n l j}\right|^{2}+\left|g_{n l j}\right|^{2}\right) V_{U}(r),
$$

where $f_{n l j}$ and $g_{n l j}$ are the upper and lower components of the Dirac wave function 3 and for the Uehling potential we use the Schwinger's parametrization ${ }^{1}$ [

$$
V_{U}(r)=\frac{\alpha}{\pi} \int_{0}^{1} d v \frac{v^{2}\left(1-v^{2} / 3\right)}{1-v^{2}}\left(-\frac{Z \alpha}{r} e^{-\lambda r}\right),
$$

with

$$
\lambda=\frac{2 m_{e}}{\sqrt{1-v^{2}}} .
$$

The exact calculation can be performed and analytic results for certain states were found in 4 . Here, at the first stage we derive a similar expression for an arbitrary state in a hydrogen-like atom. Applying the known expression for the wave function of the Dirac-Coulomb equation (see, e.g., 3. ) and integrating over coordinates, we obtain

$$
\begin{gathered}
\Delta E\left(n l_{j}\right)=-\frac{\alpha}{\pi} \eta^{2} \frac{\Gamma\left(2 \zeta+n_{r}+1\right)\left(n_{r}\right) !}{\frac{Z \alpha}{\eta}-\nu} \times \\
\sum_{i, k=0}^{n_{r}} \frac{(-1)^{i+k}}{i !\left(n_{r}-i\right) ! k !\left(n_{r}-k\right) !} \frac{\Gamma(2 \zeta+i+k)}{\Gamma(2 \zeta+i+1) \Gamma(2 \zeta+k+1)} \times \\
\left\{m\left[\left(\frac{Z \alpha}{\eta}-\nu\right)^{2}+\left(n_{r}-i\right)\left(n_{r}-k\right)\right]\right. \\
\left.-E_{n l j}\left(\frac{Z \alpha}{\eta}-\nu\right)\left(2 n_{r}-i-k\right)\right\} \times \\
\left\{K_{1,2, i+k+2 \zeta}\left(\widetilde{\kappa}_{n}\right)-\frac{1}{3} K_{2,2, i+k+2 \zeta}\left(\widetilde{\kappa}_{n}\right)\right\}
\end{gathered}
$$

where

$$
\begin{aligned}
\nu & =(-1)^{j+l+1 / 2}(j+1 / 2), \\
\zeta & =\sqrt{\nu^{2}-(Z \alpha)^{2}}, \\
\eta & =\sqrt{1-\left(E_{n l j} / m\right)^{2}}, \\
n_{r} & =n-|\nu|, \\
\widetilde{\kappa}_{n} & =n \eta \kappa_{n} /(Z \alpha),
\end{aligned}
$$

and $E_{n l j}$ is the exact relativistic energy of the $n l_{j}$ state for the Dirac-Coulomb problem.

The base integrals, defined as

$$
K_{a b c}(\kappa)=\int_{0}^{1} d v \frac{v^{2 a}}{\left(1-v^{2}\right)^{b / 2}}\left(\frac{\kappa \sqrt{1-v^{2}}}{1+\kappa \sqrt{1-v^{2}}}\right)^{c}
$$

1 Throughout the paper we apply the relativistic units in which $c=\hbar=1$. are known in a closed form [1] (cf. 4]):

$$
\begin{gathered}
K_{a b c}(\kappa)=\frac{1}{2} \kappa^{c} B\left(a+\frac{1}{2}, 1-\frac{b}{2}+\frac{c}{2}\right) \\
\times{ }_{3} F_{2}\left(\frac{c}{2}, \frac{c}{2}+\frac{1}{2}, 1-\frac{b}{2}+\frac{c}{2} ; \frac{1}{2}, a+\frac{3}{2}-\frac{b}{2}+\frac{c}{2} ; \kappa^{2}\right) \\
-\frac{c}{2} \kappa^{c+1} B\left(a+\frac{1}{2}, \frac{3}{2}-\frac{b}{2}+\frac{c}{2}\right) \\
\times{ }_{3} F_{2}\left(\frac{c}{2}+1, \frac{c}{2}+\frac{1}{2}, \frac{3}{2}-\frac{b}{2}+\frac{c}{2} ; \frac{3}{2}, a+2-\frac{b}{2}+\frac{c}{2} ; \kappa^{2}\right) .
\end{gathered}
$$

Here ${ }_{3} F_{2}(\alpha, \beta, \gamma ; \delta, \epsilon ; z)$ stands for the generalized hypergeometric function [7] and $B(\alpha, \beta)$ is the beta function.

Such expressions containing the hypergeometric function ${ }_{3} F_{2}$ are cumbersome and far from being transparent; however, various simpler asymptotic expressions are known 4,5,1. Here we intend to expand in $Z \alpha$. The leading term of the $\Delta E\left(n l_{j}\right)$ expansion in $Z \alpha$ (see the $F$ term in (1)) was studied by us in detail previously [1 (see also [5]). Now, we derive from Eq. (4) the leading relativistic correction to $\Delta E^{(0)}\left(n l_{j}\right)$, related to the $H$ term in (1).

In the limit $Z \alpha \rightarrow 0$ Eq.(7) transforms to an expression for the nonrelativistic correction to energy

$$
\begin{aligned}
\Delta E^{(\mathrm{NR})}(n l) & =-\frac{\alpha(Z \alpha)^{2}}{\pi n^{2}}(n+l) ! \sum_{i, k=0}^{n_{r}} \frac{(-1)^{i+k} n_{r} !}{i !\left(n_{r}-i\right) ! k !\left(n_{r}-k\right) !} \\
& \times \frac{(2 l+i+k+1) !}{(2 l+i+1) !(2 l+k+1) !} \\
& \times\left[K_{1,2,2 l+i+k+2}\left(\kappa_{n}\right)-\frac{1}{3} K_{2,2,2 l+i+k+2}\left(\kappa_{n}\right)\right] .
\end{aligned}
$$

This expression differs in form from those in [5] and [1, but agrees with them.

\section{Results for the low lying levels}

The explicit expression for the $H$ term is

$$
\begin{gathered}
H_{n l j}\left(\kappa_{n}\right)=\frac{\left(n_{r}\right) !(j+n+1 / 2) !}{2(2 j+1)(n-\nu)} \sum_{i, k=0}^{n_{r}} \frac{(-1)^{i+k}}{i !\left(n_{r}-i\right) ! k !\left(n_{r}-k\right) !} \\
\quad \times \frac{(2 j+i+k) !}{(2 j+i+1) !(2 j+k+1) !} \\
\times\left\{\left[2(i+k-2 \nu)+\frac{\nu(2 j+1)(4 n-2 j-i-k-1)}{n^{2}}\right.\right. \\
+(2 j+2 i-2 \nu+1)(2 j+2 k-2 \nu+1) \\
\quad \times(\psi(2 j+i+k+1)+\psi(j+n+3 / 2) \\
\left.\left.-\psi(2 j+i+2)-\psi(2 j+k+2)-\frac{(3 n-2 \nu)(2 n-2 j-1)}{4 n^{2}(n-\nu)}\right)\right]
\end{gathered}
$$


E. Yu. Korzinin, V. G. Ivanov, and S. G. Karshenboim: Vacuum polarization. .

Table 1. Asymptotics of the relativistic corrections $H_{n l j}(x)$ for some low-lying states. For $n l_{j}$ states $x=\kappa_{n}$.

$$
\begin{aligned}
& \begin{array}{ll}
\hline n l_{j} & H_{n l j}(x) \\
\hline 1 s_{1 / 2} & -\frac{1}{3} \ln (2 x)-\frac{\pi^{2}}{9}+\frac{23}{18}-\frac{1}{2 x^{2}} \\
\hline 2 s_{1 / 2} & -\frac{5}{12} \ln (2 x)-\frac{\pi^{2}}{9}+\frac{103}{72}-\frac{35}{16 x^{2}} \\
2 p_{1 / 2} & -\frac{5}{12} \ln (2 x)-\frac{\pi^{2}}{9}+\frac{361}{216}-\frac{35}{16 x^{2}} \\
\hline 3 s_{1 / 2} & -\frac{1}{3} \ln (2 x)-\frac{\pi^{2}}{9}+\frac{25}{18}-\frac{67}{18 x^{2}} \\
3 p_{1 / 2} & -\frac{1}{3} \ln (2 x)-\frac{\pi^{2}}{9}+\frac{335}{216}-\frac{67}{18 x^{2}} \\
3 d_{3 / 2} & -\frac{1}{9} \ln (2 x)-\frac{\pi^{2}}{18}+\frac{8279}{10800}-\frac{25}{18 x^{2}} \\
\hline 4 s_{1 / 2} & -\frac{13}{48} \ln (2 x)-\frac{\pi^{2}}{9}+\frac{2327}{1728}-\frac{335}{64 x^{2}} \\
4 p_{1 / 2} & -\frac{13}{48} \ln (2 x)-\frac{\pi^{2}}{9}+\frac{63287}{43200}-\frac{335}{64 x^{2}} \\
4 d_{3 / 2} & -\frac{5}{48} \ln (2 x)-\frac{\pi^{2}}{18}+\frac{16243}{21600}-\frac{35}{16 x^{2}} \\
4 f_{5 / 2} & -\frac{7}{144} \ln (2 x)-\frac{\pi^{2}}{27}+\frac{3038863}{6350400}-\frac{71}{64 x^{2}} \\
\hline
\end{array} \\
& \times\left(K_{1,2,2 j+i+k+1}\left(\kappa_{n}\right)-\frac{1}{3} K_{2,2,2 j+i+k+1}\left(\kappa_{n}\right)\right) \\
& +(2 j+2 i-2 \nu+1)(2 j+2 k-2 \nu+1) \\
& \times\left[L_{1,2,2 j+i+k+1}\left(\kappa_{n}\right)-\frac{1}{3} L_{2,2,2 j+i+k+1}\left(\kappa_{n}\right)\right. \\
& -\frac{(2 n-2 j-1)(2 j+i+k+1)}{4 n^{2} \kappa_{n}} \\
& \left.\left.\times\left(K_{1,3,2 j+i+k+2}\left(\kappa_{n}\right)-\frac{1}{3} K_{2,3,2 j+i+k+2}\left(\kappa_{n}\right)\right)\right]\right\},
\end{aligned}
$$

where

$$
L_{a b c}(\kappa)=\frac{\partial K_{a b c}(\kappa)}{\partial c} .
$$

Here $\psi(z)$ stands for the logarithmic derivative of the Euler Gamma function

$$
\begin{aligned}
\psi(n) & =\psi(1)+\sum_{k=1}^{n-1} \frac{1}{k}, \\
\psi(n+1 / 2) & =2 \psi(2 n)-\psi(n)-2 \ln 2 \\
& =\psi(1)+2 \ln 2+2 \sum_{k=0}^{n-1} \frac{1}{2 k+1},
\end{aligned}
$$

and $-\psi(1)=\mathcal{C}$ is the Euler constant, which finally cancels out in (11).

Various high- $\kappa$ asymptotics of $K_{a b c}$ were studied previously and applying (11) to the low-lying states we derive results for the relativistic correction $H_{n l j}$, which are presented in Table 1 for states with $j=l+1 / 2$. Separately we present in Table 2 the asymptotic results related to the difference $\Delta_{n l}^{\mathrm{FS}}$ defined in Eq. (3). The difference is the leading Uehling contribution to the fine structure and thus it is the part of $H_{n l j}(x)$ of most interest.
Table 2. Asymptotics of the Uehling contribution $\Delta_{n l}^{\mathrm{FS}}(x)$ at $x \gg 1$ for the fine structure of some of the lowest states. For the $n l$ states $x=\kappa_{n}$.

\begin{tabular}{ll}
\hline$n l$ & \multicolumn{1}{c}{$\Delta_{n l}^{\mathrm{FS}}(x)$} \\
\hline $2 p$ & $\frac{1}{3} \ln 2 x+\frac{\pi^{2}}{18}-\frac{215}{216}+\frac{27}{16 x^{2}}$ \\
\hline $3 p$ & $\frac{2}{9} \ln 2 x+\frac{\pi^{2}}{18}-\frac{355}{432}+\frac{7}{3 x^{2}}$ \\
$3 d$ & $\frac{2}{27} \ln 2 x+\frac{\pi^{2}}{54}-\frac{10559}{32400}+\frac{8}{9 x^{2}}$ \\
\hline $4 p$ & $\frac{1}{6} \ln 2 x+\frac{\pi^{2}}{18}-\frac{32101}{43200}+\frac{195}{64 x^{2}}$ \\
$4 d$ & $\frac{1}{18} \ln 2 x+\frac{\pi^{2}}{54}-\frac{36971}{129600}+\frac{69}{64 x^{2}}$ \\
$4 f$ & $\frac{1}{36} \ln 2 x+\frac{\pi^{2}}{108}-\frac{246373}{1587600}+\frac{39}{64 x^{2}}$ \\
\hline
\end{tabular}

\section{Results for the near circular states}

The general expression (11) contains a double summation. However, for circular $(l=n-1, j=l+1 / 2)$ and near circular states only a few terms contribute to the sum and the result is relatively simple. In the limit $\kappa_{n} \gg 1$, the result for small values of $n-l$ reads

$$
\begin{aligned}
& H_{n, n-1, n-1 / 2}\left(\kappa_{n}\right)=\frac{1}{n^{2}}\left\{\frac{1}{3}\left(-\ln \left(2 \kappa_{n}\right)+\psi(2 n)-\psi(1)\right)\right. \\
& \left.-\frac{2}{3} n \psi^{\prime}(2 n)+\frac{5}{18}-\frac{1}{2} \frac{n^{2}}{\kappa_{n}^{2}}+\mathcal{O}\left(\frac{n^{3}}{\kappa_{n}^{3}}\right)\right\}, \\
& H_{n, n-1, n-3 / 2}\left(\kappa_{n}\right)= \\
& \frac{1}{n^{2}}\left\{\frac{(n+3)}{3(n-1)}\left(-\ln \left(2 \kappa_{n}\right)+\psi(2 n)-\psi(1)\right)\right. \\
& -\frac{2 n^{2}}{3(n-1)} \psi^{\prime}(2 n)+\frac{10 n^{3}-21 n^{2}-4 n+12}{18(n-1)^{2}(2 n-1)} \\
& \left.-\frac{2 n^{3}+6 n^{2}-3 n+1}{4 n^{2}(n-1)} \frac{n^{2}}{\kappa_{n}^{2}}+\mathcal{O}\left(\frac{n^{3}}{\kappa_{n}^{3}}\right)\right\} \text {, } \\
& H_{n, n-2, n-3 / 2}\left(\kappa_{n}\right)= \\
& \frac{1}{n^{2}}\left\{\frac{n+3}{3(n-1)}\left(-\ln \left(2 \kappa_{n}\right)+\psi(2 n)-\psi(1)\right)-\frac{2 n^{2}}{3(n-1)} \psi^{\prime}(2 n)\right. \\
& +\frac{20 n^{4}-76 n^{3}+7 n^{2}+64 n-24}{18(n-1)^{2}(2 n-1)^{2}} \\
& \left.-\frac{2 n^{3}+6 n^{2}-3 n+1}{4 n^{2}(n-1)} \frac{n^{2}}{\kappa_{n}^{2}}+\mathcal{O}\left(\frac{n^{3}}{\kappa_{n}^{3}}\right)\right\} \text {, } \\
& H_{n, n-2, n-5 / 2}\left(\kappa_{n}\right)= \\
& \frac{1}{n^{2}}\left\{\frac{n+6}{3(n-2)}\left(-\ln \left(2 \kappa_{n}\right)+\psi(2 n)-\psi(1)\right)-\frac{2 n^{2}}{3(n-2)} \psi^{\prime}(2 n)\right. \\
& +\frac{40 n^{6}-316 n^{5}+510 n^{4}+565 n^{3}-1951 n^{2}+1482 n-342}{18(n-1)(n-2)^{2}(2 n-1)^{2}(2 n-3)}
\end{aligned}
$$




$$
\left.-\frac{n^{3}+6 n^{2}-6 n+4}{2 n^{2}(n-2)} \frac{n^{2}}{\kappa_{n}^{2}}+\mathcal{O}\left(\frac{n^{3}}{\kappa_{n}^{3}}\right)\right\} .
$$

The natural parameter of the expansion is $n / \kappa_{n}$, not just $1 / \kappa_{n}$, as one can observe in a limit $n \gg 1$

$$
\begin{gathered}
H_{n, n-1, j}\left(\kappa_{n}\right)=H_{n, n-2, j}\left(\kappa_{n}\right)= \\
\frac{1}{n^{2}}\left\{\frac{1}{3}\left(\ln \left(\frac{n}{\kappa_{n}}\right)-\psi(1)\right)-\frac{1}{18}-\frac{1}{2} \frac{n^{2}}{\kappa_{n}^{2}}+\ldots\right\},
\end{gathered}
$$

This parameter can be easily understood from the presentation in the coordinate space (see [1] for more discussion).

For the fine-structure splitting we find

$$
\begin{aligned}
& \Delta_{n, n-1}^{\mathrm{FS}}= \frac{1}{n^{3}}\left\{\frac{4}{3} \frac{n}{n-1}\left(\ln \left(2 \kappa_{n}\right)-\psi(2 n)+\psi(1)+\frac{n}{2} \psi^{\prime}(2 n)\right)\right. \\
&- \frac{n\left(4 n^{2}-24 n+17\right)}{18(n-1)^{2}(2 n-1)}+\frac{8 n^{2}-3 n+1}{4 n(n-1)} \frac{n^{2}}{\kappa_{n}^{2}} \\
&\left.+\mathcal{O}\left(\frac{n^{3}}{\kappa_{n}^{3}}\right)\right\}, \\
& \Delta_{n, n-2}^{\mathrm{FS}}=\frac{1}{n^{3}}\left\{\frac{4}{3} \frac{n^{2}}{(n-1)(n-2)}\right. \\
& \times\left(\ln \left(2 \kappa_{n}\right)-\psi(2 n)+\psi(1)+\frac{n}{2} \psi^{\prime}(2 n)\right)
\end{aligned}
$$$$
-\frac{n\left(16 n^{6}-424 n^{5}+1764 n^{4}-2816 n^{3}+1973 n^{2}-576 n+54\right.}{18(n-1)^{2}(n-2)^{2}(2 n-1)^{2}(2 n-3)}
$$$$
18(n-1)^{2}(n-2)^{2}(2 n-1)^{2}(2 n-3)
$$$$
\left.+\frac{8 n^{3}-9 n^{2}+13 n-6}{4 n(n-1)(n-2)} \frac{n^{2}}{\kappa_{n}^{2}}+\mathcal{O}\left(\frac{n^{3}}{\kappa_{n}^{3}}\right)\right\},
$$

and its asymptotics for $n \gg 1$ are

$$
\begin{gathered}
\Delta_{n, n-1}^{\mathrm{FS}}=\Delta_{n, n-2}^{\mathrm{FS}}= \\
\frac{1}{n^{3}}\left\{\frac{4}{3}\left(\ln \left(\frac{\kappa_{n}}{n}\right)+\psi(1)\right)+\frac{2}{9}+\frac{2 n^{2}}{\kappa_{n}^{2}}+\ldots\right\} .
\end{gathered}
$$

\section{Asymptotic behavior at low $\kappa_{n}$}

We are mostly interested in $\kappa_{n} \geq 1$ and $\kappa_{n} \gg 1$, however, for the highly excited states one can arrive at the situation when $\kappa$ is large, but $\kappa_{n}$ is small.

For the case of low $\kappa_{n}$ we obtain asymptotics of Eq. (11) for small $\kappa_{n}$

$$
\begin{aligned}
H_{n l j}\left(\kappa_{n}\right) & \approx \kappa_{n}^{2 j+1} \frac{(n+j+1 / 2) !(2 j+3) ! !}{2(2 j+1)^{3}(n-\nu)\left(n_{r}\right) !(2 j+1) !(2 j+4) ! !} \\
& \times\left\{-\frac{(2 n-2 j-1)^{2} \nu}{n^{2}}+(2 j-2 \nu+1)^{2}\right. \\
& \times\left[\ln \kappa_{n}+\frac{1}{2} \psi(j+1 / 2)+\psi(j+n+3 / 2)\right. \\
& -\psi(2 j+2)-\frac{1}{2} \psi(j+3)-\frac{1}{2 j+1}+\frac{1}{2 j+3} \\
& \left.\left.+\frac{2 j-2 n+1}{4 n^{2}}\left(2 j+3+\frac{n}{n-\nu}\right)\right]\right\} .
\end{aligned}
$$

The above asymptotics prove to be especially simple for the case $j<l$ :

$$
H_{n l j}\left(\kappa_{n}\right) \approx-\kappa_{n}^{2 j+1} \frac{(n+j+1 / 2) !(2 j+3) ! !}{n^{2}(2 j+1)^{3}\left(n_{r}-1\right) !(2 j) !(2 j+4) ! !} .
$$

In the case of low- $\kappa$ another asymptotics can also be of interest, namely a result in the leading order in $\kappa$ but exact in $(Z \alpha)$ :

$$
\begin{gathered}
\Delta E\left(n l_{j}\right) \approx-\frac{\alpha}{\pi}\left(\frac{n \eta \kappa_{n}}{Z \alpha}\right)^{2 \zeta} \frac{\zeta+1}{4 \zeta^{2}(2 \zeta+3)} \\
\frac{\eta^{2} \Gamma\left(2 \zeta+n_{r}+1\right)}{\left(n_{r}\right) !\left(\frac{Z \alpha}{\eta}-\nu\right) \Gamma(2 \zeta)} B(3 / 2, \zeta) \times \\
\times\left\{m\left[\left(\frac{Z \alpha}{\eta}-\nu\right)^{2}+n_{r}^{2}\right]-2 n_{r} E_{n l j}\left(\frac{Z \alpha}{\eta}-\nu\right)\right\} .
\end{gathered}
$$

\section{Relativistic corrections in the logarithmic approximation}

To verify our calculations we consider a limit $\ln \kappa_{n} \gg 1$ and find the logarithmic terms within the effective charge

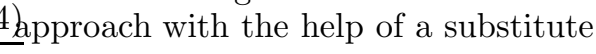

$$
Z \alpha \longrightarrow Z \alpha\left(\kappa_{n}\right)=Z \alpha\left(1+\frac{2 \alpha}{3 \pi} \ln \kappa_{n}\right)
$$

We find

$$
\begin{aligned}
H_{n l j}^{\log }\left(\kappa_{n}\right) & =-\frac{4}{3} \frac{1}{n}\left(\frac{1}{j+1 / 2}-\frac{3}{4 n}\right) \ln \kappa_{n}, \\
\Delta_{n l}^{\mathrm{FS}, \log }\left(\kappa_{n}\right) & =\frac{4}{3} \frac{1}{n} \frac{1}{l(l+1)} \ln \kappa_{n},
\end{aligned}
$$

in agreement with the direct calculations above.

\section{Corrections due to the finite nuclear mass and anomalous magnetic moment of the orbiting particle}

One feature of most exotic atoms is that a gap between the mass of the orbiting particle $m$ and of the nucleus $M$ is not so large as in a conventional atom (where $m=m_{e}$ and thus $m / M \simeq 1 /(1836 A)$, where $A$ is the mass number). As a result, various recoil effects become important. Another important property of some exotic atoms is the presence of the anomalous magnetic moment of the orbiting particle, which is not small. There are two kinds of orbiting particles with spin $1 / 2$ which can be described by the Dirac equation. While the recoil effects are important for both muonic and antiprotonic atoms, the effects of the non-zero anomalous magnetic moment take place 
only in antiprotonic atoms $\left(\kappa^{\prime}=\left(g_{p}-2\right) / 2 \simeq 1.79\right)^{2}$. The muon anomalous magnetic moment can be treated perturbatively and taken into account together with related radiative corrections.

We also note that the fine structure is considered as a structure of levels due to the interaction of the spin of the orbiting particle and the orbital moment. In this consideration we neglect the nuclear magnetic moment and thus the hyperfine effects. If the nucleus has a spin, the energy should be averaged over it to be compared to our result. Such an asymmetric treatment is reasonable when the nucleus is either spinless or heavier than the orbiting particle and cannot be applied to, e.g., protonium, a bound system of a proton and antiproton. In the case of heavy nuclei it is customary to separate the fine and hyperfine structure and for this case we consider below the recoil effects in the fine-structure splitting.

Below we take into account the finite nuclear mass and anomalous magnetic moment of the orbiting particles. To do that we re-visit the well-known leading contributions to the fine structure, found perturbatively, and show that their dependence on the nuclear mass and the anomalous magnetic moment of the orbiting particle is of a universal form. That allows us to adjust the results for $\Delta^{\mathrm{FS}}$.

Above we calculated the leading contribution to the fine splitting

$$
\Delta_{n l}^{\mathrm{FS}}\left(\kappa_{n}\right)=H_{n, l, l+1 / 2}\left(\kappa_{n}\right)-H_{n, l, l-1 / 2}\left(\kappa_{n}\right)
$$

in the external field approximation and neglecting the muon anomalous magnetic moment.

Now, we explain how to take into account the finite mass of the nucleus and its anomalous magnetic moment for the fine structure. Similar effects are also important for relativistic corrections in general; however, the general case is much more complicated and will be considered elsewhere.

The explicit results for $\Delta_{n l}^{\mathrm{FS}}\left(\kappa_{n}\right)$ were found here by applying the Dirac equation. Let us consider this particular effect perturbatively. In the case of $\kappa^{\prime}=0$ and $m / M=0$ we start from a Hamiltonian

$$
H^{0}=H_{\mathrm{NR}}^{0}+H_{\mathrm{FS}}^{0}
$$

where

$$
H_{\mathrm{NR}}^{0}=\frac{\mathbf{p}^{2}}{2 m_{R}}+V(r)
$$

is the complete nonrelativistic Hamiltonian for the problem $V(r)=V_{C}(r)+V_{U}(r), m_{R}$ is the reduced mass, which in the limit $m / M=0$ is equal to the particle mass $m$, and the fine-structure effects are described by the potential

$$
H_{\mathrm{FS}}^{0}=\frac{1}{2 m^{2}} \frac{1}{r} \frac{\partial V(r)}{\partial r}(\mathbf{s} \cdot \mathbf{l}) .
$$

2 The proton anomalous magnetic moment is customarily denoted by $\kappa$. In our paper (see, e.g., 19 ), we use $\kappa$ for the ratio $(Z \alpha m) / m_{e}$ and here we denote the proton anomalous magnetic moment by $\kappa^{\prime}$.
In terms of the eigenfunctions $\Psi_{\mathrm{NR}}^{0}$ of Hamiltonian $H_{\mathrm{NR}}^{0}$, we present the fine structure as

$$
h_{\mathrm{FS}}=\left\langle\Psi_{\mathrm{NR}}^{0}\left|H_{\mathrm{FS}}\right| \Psi_{\mathrm{NR}}^{0}\right\rangle,
$$

where $h_{\mathrm{FS}}$ is a reduced Hamiltonian for variables related to spin and angular momentum only. The complete fine structure, which is not necessarily related to the pure Coulomb problem, is eventually proportional to the matrix element of

$$
\frac{1}{2 m^{2}}\left\langle\Psi_{\mathrm{NR}}^{0}\left|\frac{1}{r} \frac{\partial V(r)}{\partial r}\right| \Psi_{\mathrm{NR}}^{0}\right\rangle(\mathbf{s} \cdot \mathbf{l}) .
$$

We note that if the potential $V(r)$ depends neither on the mass of the orbiting particle $m$ nor on the nuclear mass $M$, the wave function can depend only on the reduced mass $m_{R}$ because of the kinetic energy and on $m_{e}$ in the particular case of the Uehling potential.

Thence, from dimensional analysis we can find in the external field approximation ${ }^{3}$

$$
\frac{\alpha}{\pi}(Z \alpha)^{4} \frac{m c^{2}}{n^{2}} \Delta_{n l}^{\mathrm{FS}}\left(\kappa_{n}\right)=m G\left(m / m_{e}\right),
$$

where $G(x)$ is a dimensionless function, which depends on a state $\Psi_{\mathrm{NR}}^{0}$.

What happens if we take into account the anomalous magnetic moment and the finiteness of the nuclear mass? The nonrelativistic Hamiltonian is now of the same form as before (see (27) ) but the reduced mass

$$
m_{R}=\frac{M m}{M+m}
$$

differs from the mass of the particle $m$. Meanwhile, the Hamiltonian for the fine structure is of the form (cf., e.g., [8] for the pure Coulomb case)

$$
\begin{aligned}
& H_{\mathrm{FS}}= \frac{1}{2 m^{2}} \frac{1}{r} \frac{\partial V(r)}{\partial r}(\mathbf{s} \cdot \mathbf{l}) \cdot\left[\left(1+2 \kappa^{\prime}\right)+\frac{2 m}{M}\left(1+\kappa^{\prime}\right)\right] \\
&=\frac{m_{R}^{2}}{m^{2}}\left[\left(1+2 \kappa^{\prime}\right)+\frac{2 m}{M}\left(1+\kappa^{\prime}\right)\right] \\
& \quad \times \frac{1}{r} \frac{1}{m_{R}^{2}} \frac{\partial V(r)}{\partial r}(\mathbf{s} \cdot \mathbf{l}) .
\end{aligned}
$$

Thus, the result reads

$$
\begin{gathered}
m \Delta_{n l}^{\mathrm{FS}}\left(\kappa_{n}\right) \rightarrow \frac{m_{R}^{2}}{m^{2}}\left[\left(1+2 \kappa^{\prime}\right)+\frac{2 m}{M}\left(1+\kappa^{\prime}\right)\right] \\
\times m_{R} \Delta_{n l}^{\mathrm{FS}}\left(\kappa_{R} / n\right)
\end{gathered}
$$

and the splitting is

$$
\frac{\alpha}{\pi}(Z \alpha)^{4} \frac{m c^{2}}{n^{2}} \frac{m_{R}^{3}}{m^{3}}\left[\left(1+2 \kappa^{\prime}\right)+\frac{2 m}{M}\left(1+\kappa^{\prime}\right)\right]
$$

${ }^{3}$ A similar result takes place for the whole fine-structure splitting and for a contribution to it in any order in $\alpha$ once we consider a perturbation of the Coulomb potential by the Uehling potential. In our case, only a term linear in $\alpha$ is needed. 


$$
\times \Delta_{n l}^{\mathrm{FS}}\left(\kappa_{R} / n\right) .
$$

This result follows from dimentioanl analyses in (31) once we take into account all $m$-factors in (32). It includes the complete mass dependence and the anomalous magnetic moment and we can now use the results obtained above for the fine-structure difference ${ }^{4} \Delta_{n l}^{\mathrm{FS}}\left(\kappa_{n}\right)$.

\section{Summary}

There is a wide variety of exotic atoms, which have been or may be successfully produced; however, only in two kinds of such atoms the orbiting particle has spin $1 / 2$, namely in muonic and antiprotonic atoms. In this paper we study fine structure caused by the interaction of the spin of the orbiting particle and the orbital moment. Effects of the nuclear spin and magnetic moment are neglected. That is basically correct in two cases. Firstly, one can study an exotic atom with a spinless nucleus. Secondly, one can consider the energy averaged over the nuclear spin. The latter is correct as an approximation and the corrections enter in order $(m / M)^{2}$.

Concluding, we present here expressions for the fine splitting in both muonic and antiprotonic atoms which consist of the leading term and the Uehling correction and properly include the anomalous magnetic moment and recoil effects.

Below we summarize results known in closed analytic form for antiprotonic and muonic atoms. While the kinematic corrections discussed in the previous section are well-known, in this paper we found various presentations for the leading radiative correction $\left(\Delta_{n l}^{\mathrm{FS}}\right)$ in Sects. 3-5.

\subsection{Antiprotonic atoms}

The QED result for the fine splitting in antiprotonic atoms is of the form

$$
\begin{aligned}
\Delta E_{\mathrm{FS}}(n l) & =\frac{1}{2} \frac{(Z \alpha)^{4} m_{R} c^{2}}{n^{3}}\left(\frac{m_{R}}{m}\right)^{2} \\
& \times\left[\left(1+2 \kappa^{\prime}\right)+\frac{2 m}{M}\left(1+\kappa^{\prime}\right)\right] \\
& \times\left[\frac{1}{l(l+1)}+2 n \frac{\alpha}{\pi} \Delta_{n l}^{\mathrm{FS}}\left(\frac{\kappa_{R}}{n}\right)\right]
\end{aligned}
$$

or

$$
\begin{aligned}
\Delta E_{\mathrm{FS}}(n l) & =\frac{1}{2} \frac{(Z \alpha)^{4} m_{R} c^{2}}{n^{3}}\left(\frac{m_{R}}{m}\right)^{2} \\
& \times\left[\left(1+2 \kappa^{\prime}\right)+\frac{2 m}{M}\left(1+\kappa^{\prime}\right)\right]
\end{aligned}
$$

\footnotetext{
${ }^{4}$ We apply here the dimensional analysis only to a term linear in perturbation $V_{U}$; however, the scaling factor, which takes into account the nuclear mass and the anomalous magnetic moment of the orbiting particle, is correct in any order and for any nonrelativistic potential, as long as the latter does not explicitly depend on $m$ or $M$.
}

$$
\begin{array}{r}
\times\left[\frac{1}{l(l+1)}\left(1+\frac{8}{3} \frac{\alpha}{\pi} \ln \left(\frac{\kappa_{R}}{n}\right)\right)\right. \\
\left.+2 n \frac{\alpha}{\pi} \Delta_{n l}^{\mathrm{FS}, \mathrm{c}}\left(\frac{\kappa_{R}}{n}\right)\right]
\end{array}
$$

where we introduced

$$
\Delta_{n l}^{\mathrm{FS}, \mathrm{c}}(x)=\Delta_{n l}^{\mathrm{FS}}(x)-\Delta_{n l}^{\mathrm{FS}, \log }(x),
$$

which does not contain the leading logarithmic term (24) for high $\kappa_{n}$. The higher order corrections include a small factor which is either $\alpha$ or $(Z \alpha)^{2}$.

The QED result above does not include any nucleusrelated effects such as the finite-size effects, virtual QED annihilation effects and effects of strong interactions. The latter may be dominant for low- $l$ states. On the contrary, for higher-l states the dominant correction to the fine structure is determined by the equations above.

\subsection{Muonic atoms}

The QED result is of the form

$$
\begin{aligned}
\Delta E_{\mathrm{FS}}(n l) & =\frac{1}{2} \frac{(Z \alpha)^{4} m_{R} c^{2}}{n^{3}}\left[1-\left(\frac{m_{R}}{M}\right)^{2}+\frac{\alpha}{\pi}\left(\frac{m_{R}}{m}\right)\right] \\
& \times\left[\frac{1}{l(l+1)}+2 n \frac{\alpha}{\pi} \Delta_{n l}^{\mathrm{FS}}\left(\frac{\kappa_{R}}{n}\right)\right]
\end{aligned}
$$

or

$$
\begin{aligned}
\Delta E_{\mathrm{FS}}(n l)= & \frac{1}{2} \frac{(Z \alpha)^{4} m_{R} c^{2}}{n^{3}}\left[1-\left(\frac{m_{R}}{M}\right)^{2}+\frac{\alpha}{\pi}\left(\frac{m_{R}}{m}\right)\right] \\
\times & {\left[\frac{1}{l(l+1)}\left(1+\frac{8}{3} \frac{\alpha}{\pi} \ln \left(\frac{\kappa_{R}}{n}\right)\right)\right.} \\
& \left.+2 n \frac{\alpha}{\pi} \Delta_{n l}^{\mathrm{FS}, \mathrm{c}}\left(\frac{\kappa_{R}}{n}\right)\right]
\end{aligned}
$$

The higher order corrections include a small factor which is either $\alpha$ or $(Z \alpha)^{2}$. Some of them have been known only for a few particular cases, but not in general. The higher order effects for some particular situations are reviewed, e.g., in [10,11.

The difference with antiprotonic atoms is a perturbative treatment of the anomalous magnetic moment of the orbiting particle. The accuracy of the muonic expression is higher than in the antiprotonic case, because of lack of the direct strong interaction and smaller importance of the nuclear finite-size effects because of a larger average distance between the orbiting particle and the nucleus.

\section{Acknowledgements}

This work was supported in part by RFBR (grant \# 0602-16156) and DFG (grant GZ 436 RUS 113/769/0-2). 
E. Yu. Korzinin, V. G. Ivanov, and S. G. Karshenboim: Vacuum polarization...

\section{References}

1. S. G. Karshenboim, V. G. Ivanov, and E. Yu. Korzinin, eprint physics/0604133 Eur. Phys. J. D (2006), to be published.

2. G. E. Pustovalov. Sov. Phys. JETP 5, (1957) 1234;

D. D. Ivanenko and G. E. Pustovalov. Adv. Phys. Sci. 61, (1957) 1943.

3. L. D. Landau and E. M. Lifshitz. Course of theoretical physics. Vol. 4: V. B. Bersetetskii, E. M. Lifshitz and L.P. Pitaevskii. Quantum electrodynamics. (Pergamon Press, Oxford, 1982).

4. S. G. Karshenboim. Can. J. Phys. 76, (1998) 169; JETP 89, (1999) 850; see 9 for corrections.

5. D. Eiras and J. Soto. Phys. Lett.B. 491, (2000) 101.

6. J. Schwinger. Particles, Sources, and Fields, Vol. 2. (Perseus Book, Reading. 1998).

7. L. J. Slatter. Generalized hypergeometric functions. (Cambridge University Press, 1966).

8. H. Grotch and R. A. Hegstrom, Phys. Rev. A4, (1971) 59.

9. S. G. Karshenboim, V. G. Ivanov and V. M. Shabaev. Can. J. Phys. 79, (2001) 81; JETP 93, (2001) 477.

10. E. Borie and G. A. Rinker, Rev. Mod. Phys. 54, (1982) 67.

11. M. I. Eides, H. Grotch and V. A Shelyuto, Phys. Rep. 342, (2001) 63. 
